\title{
Editorial
}

\section{Novel Animal Model of Diabetic Complications: The SDT Rat}

Diabetes is now increasing in not only developed countries but also developing countries. Diabetes is often a difficult problem for patients because of the changes that must be made in lifestyle plus the medications that they must take, but management becomes even more difficult and costly when complications develop. In the early stage of diabetes, many diabetic patients may not pay much attention to their glycemic control. However, after diabetic complications have developed, they experience the symptoms that arise from these complications. In this stage of diabetes, glycemic control may not be sufficient to adequately treat these complications. Furthermore, aggressive glycemic control may sometimes worsen their symptoms such as early worsening of diabetic retinopathy. These diabetic complications severely affect the patient's quality of life. In order to treat diabetic complications optimally, we should clarify the pathogenic mechanisms underlying them. In order to accomplish this mission, good animal models of diabetic complication are extremely useful. Although many animal model of diabetes have been developed, there are only a few animal models that exhibit diabetic complications. In this context, a newly established non-obese type 2 model rat, the spontaneously diabetic Torii (SDT) rat, is very relevant. At first, the SDT rat was reported as an animal model of severe diabetic retinopathy resembling human diabetic retinopathy. Later, many other diabetic complications were found in this rat. The focus of the present special issue is this novel animal model of diabetic complications: the SDT rat. I hope that the topics presented in this issue will contribute to medical research into the treatment of diabetic complications.

Akihiro Kakehashi
(Guest Editor)
Jichi Medical University
Saitama Medical Center
Saitama
Japan
E-mail: kakeaki@omiya.jichi.ac.jp

(C) Akihiro Kakehashi; Licensee Bentham Open.

This is an open access article licensed under the terms of the Creative Commons Attribution Non-Commercial License (http://creativecommons.org/licenses/by-nc/3.0/) which permits unrestricted, non-commercial use, distribution and reproduction in any medium, provided the work is properly cited. 